\title{
Estimating the temporal evolution of the median floc size during flocculation in a turbulent flow: An empirical expression
}

\author{
Zhongfan ZHU ${ }^{1,}$, and Ying WANG ${ }^{2, b}$ \\ ${ }^{1}$ College of Water Sciences, Beijing Normal University, Xinjiekouwai Street 19, Beijing 100875, \\ China \\ ${ }^{2}$ Fengtai No.2 Middle School, Fengtai District, 100071, Beijing, China \\ azhuzhongfan1985@bnu.edu.cn, blinda_wang0205@126.com
}

\begin{abstract}
Keywords: Flocculation; Temporal evolution; Floc size; Turbulent flow; Empirical expression
Abstract. The Flocculation of cohesive fine-grained sediment plays an important role in the geophysical process in estuarine and coastal zones through the complete processes of sediment transport, deposition, re-suspension and consolidation. There have been many experiments studying the temporal evolution of the median floc size during flocculation. One typical trend is that the median floc size increases rapidly when the flocculation starts, and then increases slowly with flocculation time until a steady state is approached after a long flocculation time. In this study, an empirical expression used for characterizing the temporal variation of the median floc size during the flocculation process is proposed, and comparison with those published experimental results shows its validity.
\end{abstract}

\section{Introduction}

Cohesive sediment possesses complex electro-chemical and biological-chemical characteristics. Under a flow shear environment in rivers, estuaries and coastal zones, cohesive fine-grained sediment can aggregate into different-sized flocs through the collision and the bonding between primary particles and those large flocs may disaggregate into smaller flocs/primary particles [1]. Since the transport rate of cohesive sediment is a function of the floc size and the settling velocity, sediment flocculation plays an important role in the geophysical process of estuarine and coastal zones through the complete process of sediment transport, deposition, re-suspension and consolidation.

There have been many experiments studying the temporal evolution of the median floc size during flocculation (not limited to the sediment particle) [2-10]. A typical experimental result is as follows. The median floc size grew rapidly with time at the beginning of the flocculation experiment. This is a result of more productions of large flocs due to collisions and adhesions between primary particles/smaller flocs induced by the flow shear. Then, the floc size experienced a slow increase process with increasing flocculation time. This is because those large flocs possess fragile and loose structures, therefore being susceptible to the breakage induced by the flow shear. Finally, the median floc size reached an equilibrium state or steady state (i.e. the median floc size kept approximately a constant value), due to a possible balance between the shear-induced aggregation effect and the breakage effect.

The purpose of this study is to propose an empirical expression to describe the temporal variation of the median floc size during the flocculation process, and may provide the reference for the flocculation experiment.

\section{Formulation and comparison with experimental result}

Assuming that the rate of the increase of the median floc size, $d_{50}^{\prime \prime}$, is proportional to the gap between the median floc size at a certain time and the median floc size at the steady state of flocculation development, we can write a simple expression as follows: 


$$
\frac{d}{d t}\left(d_{50}^{\prime \prime}\right)=k\left\{\left(d_{50}^{\prime \prime}\right)_{s s}-d_{50}^{\prime \prime}\right\}
$$

where $\left(d_{50}^{\prime \prime}\right)_{s s}$ is the median floc size at the steady state, $k$ being a empirical constant (its dimension is [1/min] here), and $t$ is the flocculation time. Calculating the integral of Eq. (1) and using an initial condition,

$$
d_{50}^{\prime \prime}=d_{50} \text {, at } t=0
$$

where $d_{50}$ is the size of primary particle, as well as another condition,

$$
d_{50}^{\prime \prime}=\left(d_{50}^{\prime \prime}\right)_{s s}, \text { at } t \rightarrow \infty
$$

we can have

$$
d_{50}^{\prime \prime}=\left(d_{50}^{\prime \prime}\right)_{s s}-\left\{\left(d_{50}^{\prime \prime}\right)_{s s}-d_{50}\right\} \exp (-k t)
$$

In order to validate Eq. (4), we compared it with those reported experimental results. Table 1 presents some information about these experiments, including the shear-generating apparatus (as shown in the second column), material adopted for flocculation (the third column), the primary particle size (the fourth column), and the shear condition (in the fifth column) ( $\phi$ is the particle concentration, $G$ is the flow shear rate, a parameter commonly used for characterizing the flow shear condition). Fig.1 (a)-(i) shows these comparison results. For each experiment, we present the $\left(k,\left(d_{50}^{\prime \prime}\right)_{s s}\right)$ values of the fitting curve in the last column of Table 1. We can find from Fig. 1(a)-(i) that Eq. (4) agrees with experimental

\begin{tabular}{|c|c|c|c|c|c|c|}
\hline \multirow[b]{2}{*}{ References } & \multirow[b]{2}{*}{$\begin{array}{l}\text { Shear-generating } \\
\text { apparatus }\end{array}$} & \multirow[b]{2}{*}{ Material } & \multirow{2}{*}{$\begin{array}{c}d_{50} \\
(\mu m)\end{array}$} & \multirow[b]{2}{*}{ Shear condition } & \multicolumn{2}{|c|}{ Fitting coefficients } \\
\hline & & & & & $k(1 / \mathrm{min})$ & $\left(d_{50}^{\prime \prime}\right)_{s s}(\mu m)$ \\
\hline \multirow[b]{2}{*}{2} & \multirow[b]{2}{*}{ Couette chamber } & \multirow{3}{*}{$\begin{array}{c}\text { Detroit } \\
\text { River } \\
\text { sediment }\end{array}$} & \multirow[b]{2}{*}{4.00} & $\phi=1.04 * 10^{-4} ; \mathrm{G}=200 \mathrm{~s}^{-1}$ & 0.05 & 87.00 \\
\hline & & & & $\phi=1.66^{*} 10^{-3} ; \mathrm{G}=200 \mathrm{~s}^{-1}$ & 0.28 & 25.21 \\
\hline \multirow{5}{*}{3} & \multirow{5}{*}{$\begin{array}{l}\text { Couette-flow } \\
\text { system }\end{array}$} & & \multirow{4}{*}{2.17} & $\phi=5.00 * 10^{-5} ; \mathrm{G}=75 \mathrm{~s}^{-1}$ & 0.05 & 39.54 \\
\hline & & \multirow{4}{*}{$\begin{array}{l}\text { Polystyre } \\
\text { ne latex }\end{array}$} & & $\phi=5.00 * 10^{-5} ; \mathrm{G}=100 \mathrm{~s}^{-1}$ & 0.06 & 36.65 \\
\hline & & & & $\phi=5.00 * 10^{-5} ; \mathrm{G}=125 \mathrm{~s}^{-1}$ & 0.06 & 26.52 \\
\hline & & & & $\phi=5.00 * 10^{-5} ; \mathrm{G}=150 \mathrm{~s}^{-1}$ & 0.08 & 14.47 \\
\hline & & & & $\phi=2.10^{*} 10^{-5} ; \mathrm{G}=63 \mathrm{~s}^{-1}$ & & \\
\hline \multirow{4}{*}{4} & \multirow{4}{*}{$\begin{array}{l}\text { Baffled stirred } \\
\text { tank }\end{array}$} & \multirow{4}{*}{$\begin{array}{l}\text { Polystyre } \\
\text { ne particle }\end{array}$} & \multirow{4}{*}{0.87} & $\begin{array}{l}\text { Alum concentration: } 4.3 \\
\text { mg/litre } \\
\phi=2.10^{*} 10^{-5} ; \mathrm{G}=63 \mathrm{~s}^{-1}\end{array}$ & 0.10 & 13.54 \\
\hline & & & & $\begin{array}{l}\text { Alum concentration: } 10.7 \\
\text { mg/litre } \\
\phi=2.10^{*} 10^{-5} ; \mathrm{G}=63 \mathrm{~s}^{-1}\end{array}$ & 0.30 & 41.90 \\
\hline & & & & $\begin{array}{l}\text { Alum concentration: } 32 \\
\text { mg/litre } \\
\phi=2.10^{*} 10^{-5} ; \mathrm{G}=95 \mathrm{~s}^{-1}\end{array}$ & 0.51 & 84.20 \\
\hline & & & & $\begin{array}{l}\text { Alum concentration: } 32 \\
\text { mg/litre }\end{array}$ & 0.19 & 67.01 \\
\hline \multirow{3}{*}{5} & \multirow{3}{*}{$\begin{array}{l}\text { Couette-flow } \\
\text { system }\end{array}$} & \multirow{3}{*}{$\begin{array}{l}\text { Latex } \\
\text { particle }\end{array}$} & \multirow{3}{*}{2.00} & $\phi=2.50 * 10^{-5} ; \mathrm{G}=25 \mathrm{~s}^{-1}$ & 0.01 & 46.06 \\
\hline & & & & $\phi=2.50 * 10^{-5} ; \mathrm{G}=50 \mathrm{~s}^{-1}$ & 0.03 & 38.84 \\
\hline & & & & $\phi=2.50 * 10^{-5} ; \mathrm{G}=90 \mathrm{~s}^{-1}$ & 0.05 & 30.00 \\
\hline & & & & 103 & & \\
\hline
\end{tabular}
results well as long as two fitting coefficients $\left(k,\left(d_{50}^{\prime \prime}\right)_{s s}\right)$ are determined, although some scatter can be present, which may show the validity of Eq. (4) to a certain degree.

Table 1. Information about the flocculation process in some reported experiments. 
6

$\begin{array}{cc}\begin{array}{c}\text { Couette-flow } \\ \text { system }\end{array} & \begin{array}{c}\text { Latex } \\ \text { particle }\end{array}\end{array}$

7

Baffled batch Activated vessel sludge

8

Couette-flow
system

9

An annular flume

10

Flask shaking table
Hay River

sediment

Polystyre

Polystyre ne latex particle

0.81 ne latex particle

2.10 $\phi=2.50 * 10^{-5} ; \mathrm{G}=135 \mathrm{~s}^{-1}$

0.10

19.87

$\phi=2.50 * 10^{-5} ; \mathrm{G}=195 \mathrm{~s}^{-1}$

0.10

11.74

$\phi=5.00 * 10^{-5} ; \mathrm{G}=25 \mathrm{~s}^{-1}$

0.02

41.36

2.00

$\phi=5.00 * 10^{-5} ; \mathrm{G}=32 \mathrm{~s}^{-1}$

0.03

37.73

$\phi=5.00 * 10^{-5} ; \mathrm{G}=50 \mathrm{~s}^{-1}$

0.03

35.23

$\phi=0.05 ; \mathrm{G}=19.40 \mathrm{~s}^{-1}$

0.08

121.27

$\phi=0.05 ; \mathrm{G}=37.00 \mathrm{~s}^{-1}$

0.10

100.56

$\phi=0.05 ; \mathrm{G}=113.00 \mathrm{~s}^{-1}$

0.11

58.66

$\phi=0.05 ; \mathrm{G}=346.00 \mathrm{~s}^{-1}$

0.12

25.14

$\phi=3.76^{*} 10^{-5} ; \mathrm{G}=$ $64.00 \mathrm{~s}^{-1}$

$0.01 * \mathrm{~T}^{0.45}$

70.94

$\phi=3.76^{*} 10^{-5} ; \mathrm{G}=$

$100.00 \mathrm{~s}^{-1}$

$0.02 * \mathrm{~T}^{0.45}$

67.76

$\phi=3.76^{*} 10^{-5} ; \mathrm{G}=$

$$
246.00 \mathrm{~s}^{-1}
$$

$0.01 * \mathrm{~T}^{0.45}$

38.07

Bed shear stress $=0.123$

$$
\mathrm{Pa}
$$

0.03

128.97

Bed shear stress $=0.212$

$$
\mathrm{Pa}
$$

0.09

178.10

Bed shear stress $=0.323$

$\mathrm{Pa}$

0.17

161.84

$\phi=2.00 * 10-5 ; \mathrm{G}=$ $0.45 \mathrm{~s}^{-1}$

$0.22 * 10^{-3}$

7.88

$\phi=2.00 * 10^{-5} ; \mathrm{G}=0.75 \mathrm{~s}^{-1}$

$0.32 * 10^{-3}$

9.34

$\phi=2.00 * 10^{-5} ; \mathrm{G}=0.96 \mathrm{~s}^{-1}$

$0.41 * 10^{-3}$

9.05

$\phi=2.00 * 10^{-5} ; \mathrm{G}=1.41 \mathrm{~s}^{-1}$

$0.52 * 10^{-3}$

9.68

$\phi=2.00 * 10^{-5} ; \mathrm{G}=2.40 \mathrm{~s}^{-1}$
10.42

Caption: The symbol "*" shows that the median size of the flocs is $15 \mu \mathrm{m}$, when the experiment was initiated (the experiment did not start with the primary particle). In Selomulya et al. (2002)' study [8], the flocculation time, $t$, has been incorporated into an non-dimensional parameter, and all quantities in this parameter can be found in their study except for the absolute temperature, T, when we calculated the $k$ value.

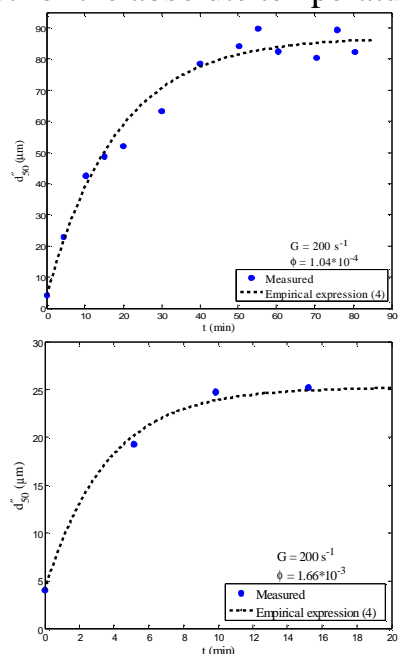

(a)

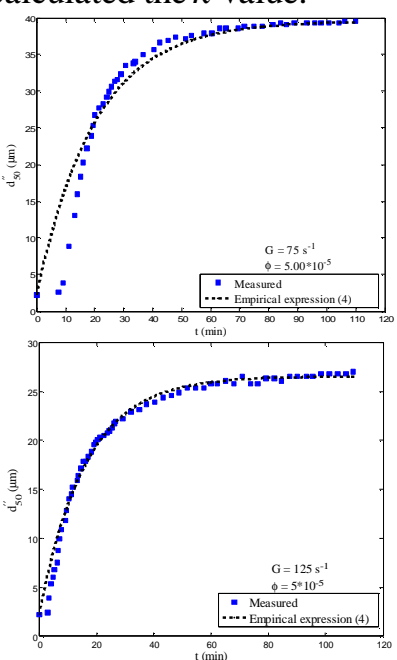

(b)

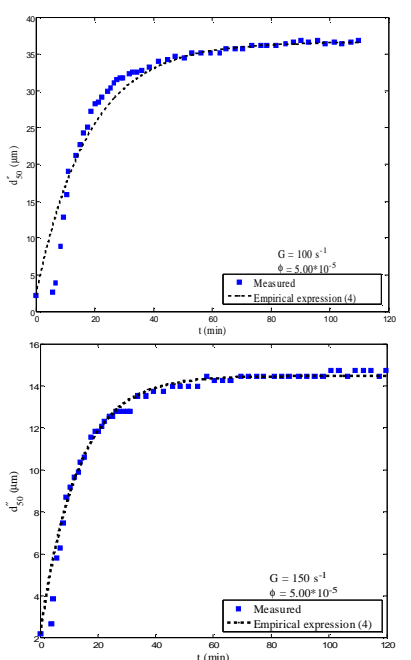



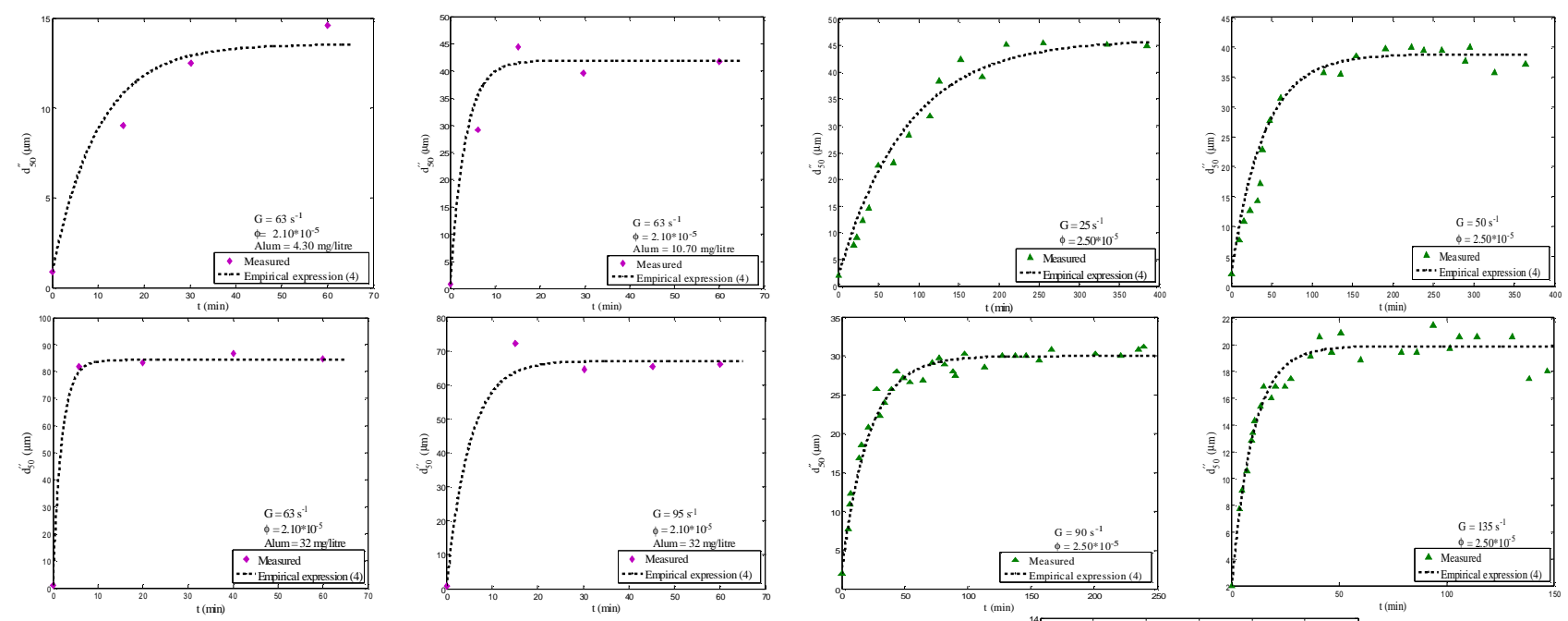

(c)

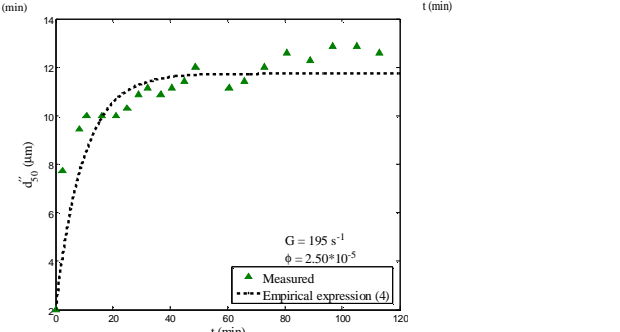

(d)
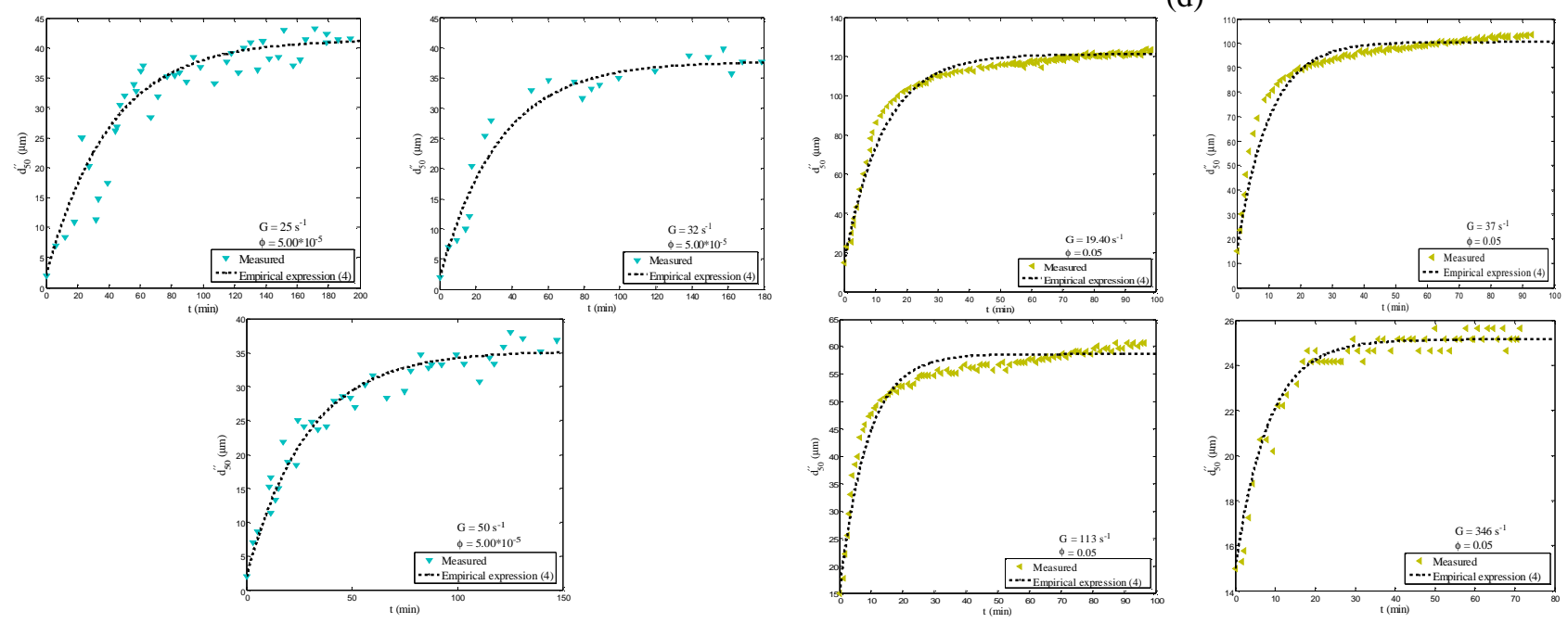

(e)

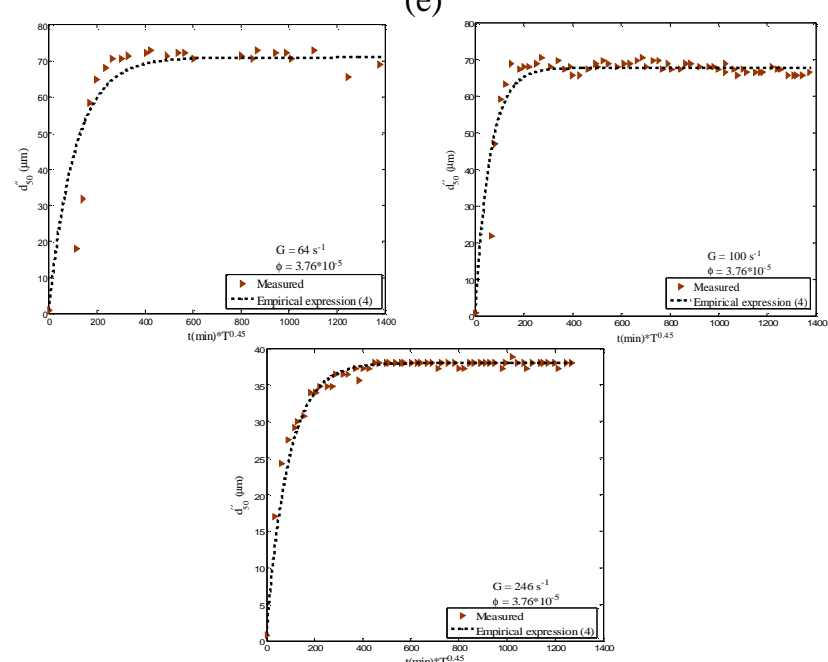

(g)

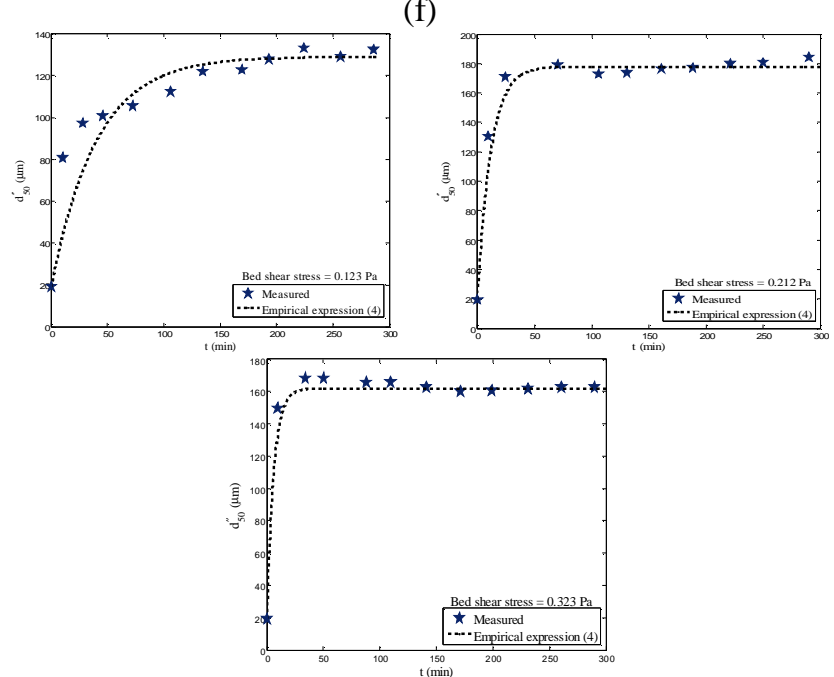

(h) 

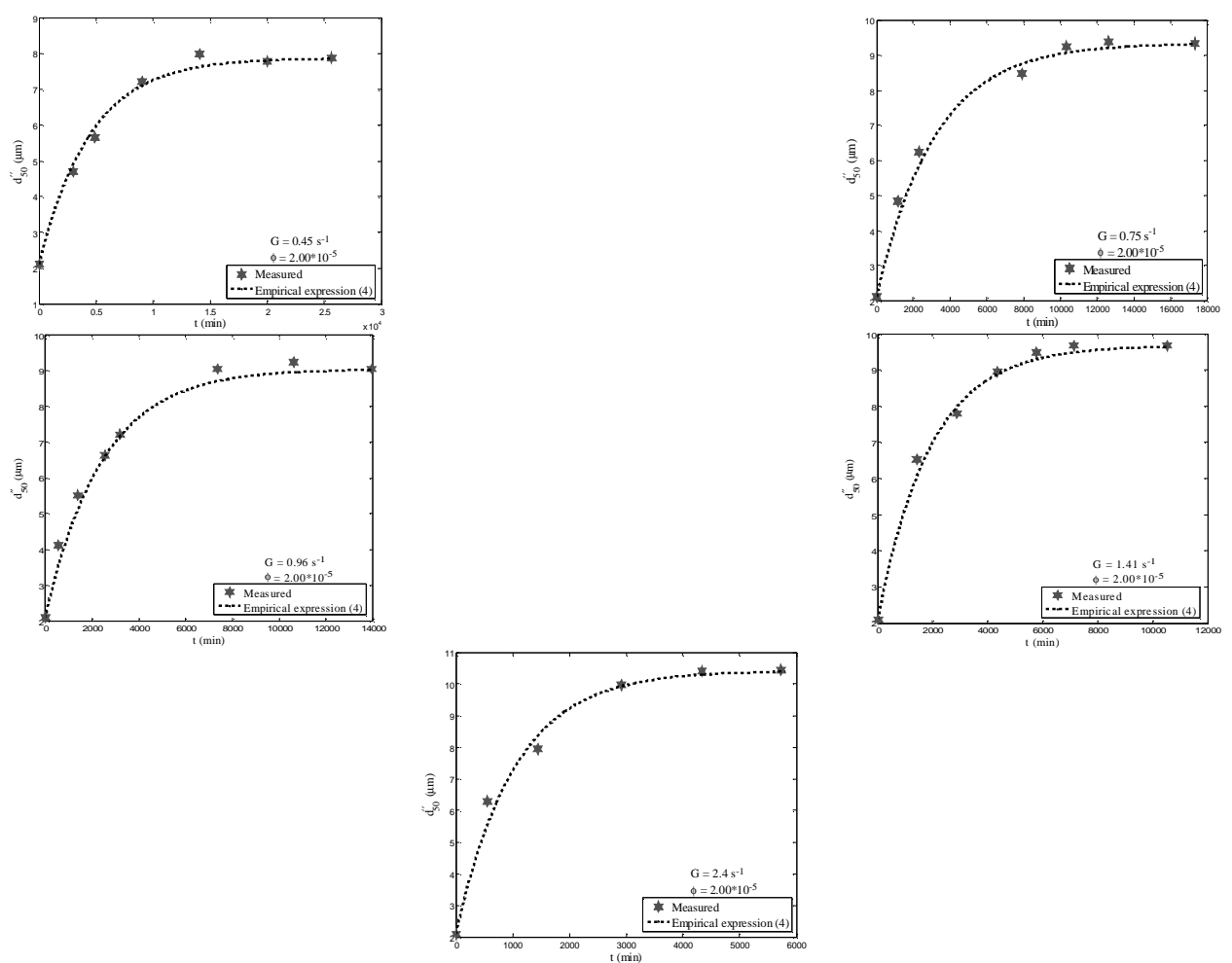

(i)

Fig. 1. Comparisons of Eq. (4) and some reported experimental data. They are from (a) Burban et al. (1989) [2], (b) Oles (1992) [3], (c) Spicer and Pratsinis (1996) [4], (d) Serra et al. (1997) [5] (e) Serra and Casamitjana (1998) [6] (f) Biggs and Lant (2000) [7] (g) Selomulya et al. (2002) [8](in this figure, T is the absolute temperature, which was not yet provided in their study), (h) Stone and Krishnappan (2003) [9] and (i) Colomer et al. (2005) [10]

\section{Concluding remarks}

Many experiments have been performed to study the temporal evolution of the median floc size during flocculation. One typical experimental result is that the median floc size increases rapidly when the flocculation starts, and then increases slowly with flocculation time until a steady state is reached after a long flocculation time. In this study, an empirical expression used for characterizing the temporal variation of the median floc size during the flocculation process is proposed, and comparison with those published experimental results shows its validity.

\section{References}

[1] M. Son and T.J. Hsu: Environ. Fluid Mech. Vol. 8 (2008), p.55

[2] P.Y. Burban, W. Lick, J. Lick: J. Geophys. Res. Vol. 94 (1989), p.8323

[3] V.Oles: J. Colloid Interface Sci. Vol. 154 (1992), p.351

[4] P.T. Spicer and S.E. Pratsinis: Water Res. Vol. 30 (1996), p.1049

[5] T. Serra, J. Colomer, X. Casamitjana: J. Colloid Interface Sci. Vol. 187 (1997), p.466

[6] T. Serra and X. Casamitjana: J. Colloid Interface Sci. Vol. 206 (1998), p.505

[7] C. Biggs and P. Lant: Water Res. Vol.34 (2000), p.2542

[8] C. Selomulya, G. Bushell, R. Amal, T.D. Waite: Langmuir Vol. 18 (2002), p.1974

[9] M.Stone and B. Krishnappan: Water Res. Vol. 37 (2003), p. 2739

[10] J. Colomer, F. Peters, C. Marrasé: Water Res. Vol. 39 (2005), p.2994 